\title{
Short communication: Genomic evaluations of final score for US Holsteins benefit from the inclusion of genotypes on cows
}

\author{
S. Tsuruta,${ }^{* 1}$ I. Misztal, ${ }^{*}$ and T. J. Lawlor† \\ *Animal and Dairy Science Department, University of Georgia, Athens 30602 \\ †Holstein Association USA Inc., Brattleboro, VT 05301
}

\section{ABSTRACT}

Currently, the US Department of Agriculture Animal Improvement Programs Laboratory utilizes a multistep procedure in genomic evaluations for US Holstein bulls and cows, with adjustments for cows. We used a single-step procedure to investigate whether adding cows' genotypes could increase reliability in genomic breeding values for bulls while minimizing bias. The first data set to 2007 was used to calculate genomic estimated breeding values (GEBV) for animals, including young genotyped bulls with no daughters and young cows (heifers) with no records in 2007. The second data set to 2011 was used to calculate GEBV for the same animals, including those young bulls with daughters and young cows with records in 2011. Genotypes $(42,503$ single nucleotide polymorphism markers) for 34,506 bulls and 5,235 cows from 356,413 bulls and 9,245,619 cows in pedigree were used to calculate single-step GEBV (ssGEBV) and multi-step GEBV (msGEBV). Regression coefficients of 2007 GEBV on 2011 progeny deviations and coefficients of determination were used as indicators of bias and reliability in 2007 GEBV for bulls with no daughters and for cows with no records in 2007, using bull genotypes only and using bull and cow genotypes. Parent averages were also calculated from estimated breeding values of parents to compare with GEBV. For genotyped bulls, inflation was larger for ssGEBV than for msGEBV, whereas reliability was higher for ssGEBV. Using all genotyped bulls and cows, reliabilities were increased by 2 to $3 \%$. Use of genotypes of high-profile cows improves reliability in ssGEBV and msGEBV for bulls.

Key words: genomic evaluation, final score, US Holstein

Received October 15, 2012.

Accepted January 15, 2013

${ }^{1}$ Corresponding author: shogo@uga.edu

\section{Short Communication}

Currently, national genomic evaluations conducted by the US Department of Agriculture Animal Improvement Programs Laboratory (USDA-AIPL, Beltsville, MD) utilize genotypes of bulls and cows. Adding genotypes of cows to a national genomic evaluation should increase the reliability of all genomic EBV (GEBV) of bulls and cows and should partially account for selection bias due to selective genotyping. With a multi-step procedure (msGBLUP) by VanRaden (2008), Schenkel et al. (2009) and VanRaden et al. (2009) reported that SNP markers could increase reliability of multistep genomic predictions (msGEBV) in Canadian Holsteins and US Holsteins, respectively. Aguilar et al. (2010) and Tsuruta et al. (2011) also showed an increase in accuracy of single-step genomic evaluations (ssGEBV) for US Holsteins. In the United States, since the first unofficial genomic evaluations were released in 2008, several thousands of animals (bulls and cows) have been genotyped every year (Wiggans et al., 2011b). Wiggans et al. (2011a) found that, with msGBLUP, the addition of genotypes of cows resulted in a decrease in the reliabilities of the genomic evaluations for bulls. The belief for this decrease in accuracy was that many of the cows that were selected for genotyping would have been selected based upon their high genetic merit or potential for a high genetic evaluation, and may have been exposed to preferential treatment, either consciously or subconsciously. To circumvent this problem, a preadjustment of the cows' traditional PTA was implemented to make the cow's PTA distribution comparable to that of the bull's PTA (Wiggans et al., 2012). It is unclear whether this preadjustment of cow PTA is optimum or if modifying some other approximation used in msGBLUP would have been more appropriate (Aguilar et al., 2010). The inclusion of genotyped cows into the genomic evaluations is viewed as desirable as they are an important part of the data associated with the selection decision, and they can contribute to the size of the reference population. These features should lead to reduced bias and additional accuracy. The single-step procedure (ssGBLUP) may provide 
us with a better theoretical and practical approach for the inclusion of cow genotypes without the need for heuristic adjustments, as with msGBLUP. It is of interest to see whether ssGBLUP could use cow genotypes for additional accuracy and reduced bias without additional heuristics.

The objective of this study was to compare changes in accuracy and potential bias in GEBV by ssGBLUP from adding genomic information on bulls and cows for final score of US Holsteins. Final scores provided by Holstein Association USA Inc. (Brattleboro, VT) were used in this analysis. We prepared 2 data sets as follows: (1) the first data set to 2007, containing 10,167,604 phenotypes for 6,012,441 cows; and (2) the second dataset to 2011 , containing $10,944,571$ phenotypes for $6,586,605$ cows.

The first data set was used to calculate GEBV for animals including young genotyped bulls with no daughters and young cows (heifers) with no records in 2007. The second data set was used to calculate GEBV for the same animals, including those young bulls with daughters and young cows with records in 2011. The animal model in national evaluation described in Tsuruta et al. (2002) was used for genomic evaluation, including genotypes of bulls and cows. In the genomic evaluation, the inverse of matrix $\mathbf{H}\left(\mathbf{H}^{-1}\right)$ replaced the inverse of matrix $\mathbf{A}\left(\mathbf{A}^{-1}\right)$ in the following mixed model equation:

$$
\left[\begin{array}{ccc}
\mathbf{X}^{\prime} \mathbf{X} & \mathbf{X}^{\prime} \mathbf{Z} & \mathbf{X}^{\prime} \mathbf{W} \\
\mathbf{Z}^{\prime} \mathbf{X} & \mathbf{Z}^{\prime} \mathbf{Z}+\mathbf{H}^{-1} \alpha_{1} & \mathbf{Z}^{\prime} \mathbf{W} \\
\mathbf{W}^{\prime} \mathbf{X} & \mathbf{W}^{\prime} \mathbf{Z} & \mathbf{W}^{\prime} \mathbf{W}+\mathbf{I} \alpha_{2}
\end{array}\right]\left[\begin{array}{l}
\boldsymbol{\beta} \\
\mathbf{a} \\
\mathbf{p}
\end{array}\right]=\left[\begin{array}{c}
\mathbf{X}^{\prime} \mathbf{y} \\
\mathbf{Z}^{\prime} \mathbf{y} \\
\mathbf{W}^{\prime} \mathbf{y}
\end{array}\right]
$$

where $\boldsymbol{\beta}=$ fixed effects; $\mathbf{a}=$ random additive genetic effects; $\mathbf{p}=$ random permanent environmental effects; and $\mathbf{X}, \mathbf{Z}, \mathbf{W}=$ incidence matrices for $\boldsymbol{\beta}, \mathbf{a}$, and $\mathbf{p}$, respectively; and

$$
\begin{gathered}
\alpha_{1}=\frac{\sigma_{e}^{2}}{\sigma_{a}^{2}}, \\
\alpha_{2}=\frac{\sigma_{e}^{2}}{\sigma_{p}^{2}} \text {, and } \\
\mathbf{H}^{-1}=\left[\begin{array}{ll}
\mathbf{A}^{11} & \mathbf{A}^{12} \\
\mathbf{A}^{21} & \mathbf{A}^{22}+\mathbf{G}^{-1}-\omega \mathbf{A}_{22}^{-1}
\end{array}\right],
\end{gathered}
$$

where $\sigma_{e}^{2}, \sigma_{a}^{2}$, and $\sigma_{p}^{2}$ are residual, additive genetic, and permanent environmental variances, respectively; $\mathbf{A}^{11}$ and $\mathbf{A}_{22}^{-1}$ are inverses of pedigree-based numerator rela- tionship matrices $\mathbf{A}_{11}$ and $\mathbf{A}_{22}$ for nongenotyped and genotyped animals, respectively; and $\mathbf{G}^{-1}$ is an inverse of a genomic relationship matrix. Weight factors $\omega=$ 0.7 and $\omega=0.5$ for $\mathbf{A}_{22}^{-1}$ were used to reduce bias in ssGEBV. Misztal et al. (2010) and Tsuruta et al. (2011) reported that bias in ssGEBV could be reduced by using a weight smaller than unity on $\mathbf{A}_{22}^{-1}$ with no significant reduction in $\mathrm{R}^{2}$. The BLUP90IOD program (Tsuruta et al., 2001) was used to calculate EBV and ssGEBV, setting a convergence criterion to $10^{-14}$, which is defined as described in Tsuruta et al. (2011). An msGBLUP (VanRaden, 2008) was also used to calculate msGEBV.

The pedigree file contained 9,602,032 animals (356,413 bulls and 9,245,619 cows), which were used to construct the numerator relationship matrix. A total of 39,741 genotyped animals (34,506 bulls and 5,235 cows) with 42,503 SNP markers from the BovineSNP50 (50K) BeadChip (Illumina Inc., San Diego, CA) were used to construct the genomic relationship matrix. The present paper addresses the potential benefit of adding genotypes on cows, especially those of high genetic merit, who may have been exposed to preferential treatment. During the period of this study, most potential bull mothers would have been required to obtain a final score as part of the selection process or for marketing purposes. Higher genetic merit animals would be more likely to be genotyped with a high-density bead chip. To investigate the effect of including genotypes on high-genetic-merit cows while keeping the genomic relationship matrix to a manageable size, genotypes from a low-density Bovine3K BeadChip (Illumina Inc.) or from cows without a phenotypic record were excluded. Regression coefficients of 2007 GEBV on deregressed evaluations for the 2011 data, and coefficients of determination $\left(\mathrm{R}^{2}\right)$ were used as indicators of bias and realized reliability accuracy of GEBV for the 2007 data, respectively (VanRaden et al., 2009). The regression coefficient should be close to unity when there is no bias in 2007 GEBV. When a regression coefficient is less than unity, the GEBV are inflated or overestimated in 2007. Parent averages (PA) were calculated from parents' EBV in 2007 with no genomic information to compare with GEBV.

Table 1 shows the computing performance for traditional (BLUP) and ssGBLUP evaluations using 2007 and 2011 data sets. Numbers of rounds at convergence and the computing time for the 2007 data set were twice as large for the 2011 data set. Use of the same pedigree and genomic information with the same unknown parent groups for 2007 and 2011 data sets may have caused slower convergence for the 2007 data set (i.e., more genotyped animals with no progenies or with 
Table 1. Computing performance at convergence for traditional (BLUP) and single-step genomic (ssGBLUP) evaluations using weights $\omega=0.7$ (upper) and $\omega=0.5$ (lower) for $\mathbf{A}_{22}^{-1}$

\begin{tabular}{|c|c|c|c|c|}
\hline \multirow[b]{2}{*}{ Model } & \multicolumn{2}{|c|}{ Number of rounds } & \multicolumn{2}{|c|}{ Time (min) } \\
\hline & 2007 data & 2011 data & 2007 data & 2011 data \\
\hline $\begin{array}{l}\text { BLUP } \\
\text { ssGBLUP }\end{array}$ & 545 & 233 & 74 & 38 \\
\hline All genotyped bulls & 581 & 371 & 168 & 126 \\
\hline & 550 & 345 & 137 & 100 \\
\hline All genotyped animals & 617 & 420 & 212 & 172 \\
\hline & 569 & 353 & 162 & 120 \\
\hline
\end{tabular}

no records were included in the 2007 data set). When using pedigree information for all animals and genotypes for only bulls and $\omega=0.7$, numbers of rounds were 7 and $60 \%$ larger, whereas computing times were 2 and 3 times longer for ssGBLUP than for BLUP in the 2007 and 2011 data sets, respectively. When using cows' genotypes in addition to bulls' genotypes, numbers of rounds were similar to those when using bulls' genotypes, but computing times were 3 to 5 times longer. With $\omega=0.5$, number of rounds were 6 to $19 \%$ smaller and computing times were 19 to $31 \%$ less than those with $\omega=0.7$.

Table 2 shows regression coefficients and $\mathrm{R}^{2}$ for $\mathrm{PA}$, ssGEBV, and msGEBV with $\omega=0.7$ when only genotypes of bulls or all genotypes of bulls and cows were used. In calculations of regression coefficients and $\mathrm{R}^{2}$, GEBV for genotyped bulls that were born after 2003 and had no daughters in 2007 and then had at least 1,10 , and 30 daughters in 2011 were used; numbers of those genotyped bulls were 3,033, 2,376, and 1,851, respectively. Regression coefficients ranged from 0.779 to 0.784 , from 0.804 to 0.814 , and from 0.996 to 1.060 for PA, ssGEBV, and msGEBV, respectively, implying that PA and ssGEBV in 2007 were inflated but msGEBV were less biased. For bulls, biases in PA were larger than those in ssGEBV; therefore, they should be reduced to improve ssGEBV. When only genotyped bulls were used, $\mathrm{R}^{2}$ in ssGEBV and msGEBV were 39.7 to $47.2 \%$ and 14.7 to $32.8 \%$ higher than those in PA, respectively. When cow genotypes were added, reliabilities in 2007 ssGEBV and msGEBV for bulls were increased by 1.9 to $2.7 \%$ and 1.3 to $3.9 \%$ from those using only bull genotypes, respectively, and the biases were reduced, but not significantly. For 2,698 young cows that were born after 2005, had no records in 2007, and had at least one record in 2011, regression coefficients showed a slight overestimation for PA and ssGEBV and an underestimation for msGEBV. For genotyped cows, $\mathrm{R}^{2}$ in ssGEBV and msGEBV were 37.6 and $42.3 \%$ higher than those in PA, respectively. Using $\omega=0.5$, Table 2 also shows the same statistics as $\omega=0.7$. For bulls, ssGEBV were less biased than those with $\omega=$

Table 2. Regression coefficients $(\delta)$ and coefficients of determination $\left(\mathrm{R}^{2}\right)$ of deregressed evaluations in 2011 on parent average (PA), single-step genomic EBV (ssGEBV) using weights $\omega=0.7$ and $\omega=0.5$ for $\mathbf{A}_{22}^{-1}$, and multi-step genomic EBV (msGEBV) in 2007 for genotyped bulls and cows using only bull genotypes and using bull and cow genotypes (upper: at least 1 daughter; middle: at least 10 daughters; lower: at least 30 daughters for each bull evaluation)

\begin{tabular}{|c|c|c|c|c|c|c|}
\hline \multirow[b]{3}{*}{ Evaluation } & \multicolumn{2}{|c|}{ Using only genotyped bulls } & \multicolumn{4}{|c|}{ Using all genotyped animals } \\
\hline & \multicolumn{2}{|c|}{ Bulls } & \multicolumn{2}{|c|}{ Bulls } & \multicolumn{2}{|c|}{ Cows } \\
\hline & $\delta$ & $\mathrm{R}^{2}$ & $\delta$ & $\mathrm{R}^{2}$ & $\delta$ & $\mathrm{R}^{2}$ \\
\hline \multirow[t]{3}{*}{$\mathrm{PA}$} & 0.784 & 0.116 & 0.785 & 0.116 & & \\
\hline & 0.779 & 0.229 & 0.780 & 0.230 & 0.920 & 0.170 \\
\hline & 0.783 & 0.265 & 0.786 & 0.267 & & \\
\hline \multirow{4}{*}{$\begin{array}{c}\text { ssGEBV } \\
\omega=0.7\end{array}$} & & & & & & \\
\hline & 0.806 & 0.162 & 0.809 & 0.165 & & \\
\hline & 0.814 & 0.337 & 0.820 & 0.346 & 0.960 & 0.234 \\
\hline & 0.804 & 0.387 & 0.813 & 0.397 & & \\
\hline \multirow[t]{3}{*}{$\omega=0.5$} & 0.936 & 0.153 & 0.944 & 0.156 & & \\
\hline & 0.949 & 0.319 & 0.959 & 0.328 & 1.171 & 0.215 \\
\hline & 0.935 & 0.357 & 0.949 & 0.368 & & \\
\hline \multirow[t]{3}{*}{ msGEBV } & 1.060 & 0.154 & 0.990 & 0.156 & & \\
\hline & 0.967 & 0.263 & 0.912 & 0.271 & 1.241 & 0.242 \\
\hline & 0.966 & 0.304 & 0.915 & 0.316 & & \\
\hline
\end{tabular}


0.7, but ssGEBV for cows were underestimated. For bulls, reliabilities were decreased by 5 to $8 \%$ for bulls and $9 \%$ for cows.

Only selected females were used in this study; the results may be different if more genotyped cows are included, depending on a trait in US Holsteins. At this time, the number of available genotypes for US Holsteins exceeds the capability of ssGBLUP as used in this study. However, refinements to ssGBLUP for a higher number of genotypes are being investigated (VanRaden et al., 2011; Legarra and Ducrocq, 2012), and predictions for young animals can be obtained indirectly (Misztal et al., 2012). Gains in reliabilities from including low-accuracy animals in genetic evaluations are expected to be minimal (Misztal et al., 2013). However, their inclusion in genetic evaluations will become necessary to control preselection bias (Patry and Ducrocq, 2011) resulting from a large number of animals that are being genomically screened as a criterion for entering into a performance recording program or to be used for breeding purposes.

\section{ACKNOWLEDGMENTS}

This study was partially funded by the Holstein Association USA Inc. (Brattleboro, VT) and by AFRI grants 2009-65205-05665 and 2010-65205-20366 from the USDA NIFA Animal Genome Program.

\section{REFERENCES}

Aguilar, I., I. Misztal, D. L. Johnson, A. Legarra, S. Tsuruta, and T. J. Lawlor. 2010. Hot topic: A unified approach to utilize phenotypic, full pedigree, and genomic information for genetic evaluation of Holstein final score. J. Dairy Sci. 93:743-752.

Legarra, A., and V. Ducrocq. 2012. Computational strategies for national integration of phenotypic, genomic, and pedigree data in a single-step best linear unbiased prediction. J. Dairy Sci. 95:46294645 .

Misztal, I., I. Aguilar, A. Legarra, and T. J. Lawlor. 2010. Choice of parameters for single-step genomic evaluation for type. J. Dairy Sci. 93(Suppl. 1):166. (Abstr.)

Misztal, I., I. Aguilar, A. Legarra, S. Tsuruta, and H. Wang. 2012. Adaptation of BGF90 package for genomic computations. J. Dairy Sci. 95(Suppl. 2):446. (Abstr.)

Misztal, I., S. Tsuruta, I. Aguilar, A. Legarra, P. M. VanRaden, and T. J. Lawlor. 2013. Methods to approximate reliabilities in single-step genomic evaluation. J. Dairy Sci. 96:647-654.

Patry, C., and V. Ducrocq. 2011. Evidence of biases in genetic evaluations due to genomic preselection in dairy cattle. J. Dairy Sci. 94:1011-1020.

Schenkel, F. S., M. Sargolzaei, G. Kistemaker, G. B. Jansen, P. Sullivan, B. J. Van Doormaal, P. M. VanRaden, and G. R. Wiggans. 2009. Reliability of genomic evaluation of Holstein cattle in Canada. Interbull Bull. 39:51-58.

Tsuruta, S., I. Misztal, I. Aguilar, and T. J. Lawlor. 2011. Multipletrait genomic evaluation of linear type traits using genomic and phenotypic data in Holsteins. J. Dairy Sci. 94:4198-4204.

Tsuruta, S., I. Misztal, L. Klei, and T. J. Lawlor. 2002. Analysis of agespecific predicted transmitting abilities for final scores in Holsteins with a random regression model. J. Dairy Sci. 85:1324-1330.

Tsuruta, S., I. Misztal, and I. Stranden. 2001. Use of the preconditioned conjugate gradient algorithm as a generic solver for mixed model equations in animal breeding applications. J. Anim. Sci. 79:1166-1172.

VanRaden, P. M. 2008. Efficient methods to compute genomic predictions. J. Dairy Sci. 91:4414-4423.

VanRaden, P. M., J. R. O'Connell, G. R. Wiggans, and K. A. Weigel. 2011. Genomic evaluations with many more genotypes. Genet. Sel. Evol. 43:10.

VanRaden, P. M., C. P. Van Tassell, G. R. Wiggans, T. S. Sonstegard, R. D. Schnabel, J. F. Taylor, and F. S. Schenkel. 2009. Invited review: Reliability of genomic predictions for North American Holstein bulls. J. Dairy Sci. 92:16-24.

Wiggans, G. R., T. A. Cooper, P. M. VanRaden, and J. B. Cole. 2011a. Technical note: Adjustment of traditional cow evaluations to improve accuracy of genomic predictions. J. Dairy Sci. 94:6188-6193.

Wiggans, G. R., P. M. VanRaden, and T. A. Cooper. 2011b. The genomic evaluation system in the United States: Past, present, future. J. Dairy Sci. 94:3202-3211.

Wiggans, G. R., P. M. VanRaden, and T. A. Cooper. 2012. Technical note: Adjustment of all cow evaluations for yield traits to be comparable with bull evaluations. J. Dairy Sci. 95:3444-3447. 